$\mathrm{E}$ EVALUAR
2017, Vol. 17, No 2.

ISSN $1667-4545$

Recuperado de https://revistas.unc.edu.ar/index.php/revaluar

Laboratorio de Evaluación Psicológica y Educativa

Facultad de Psicología - Universidad Nacional de Córdoba

\title{
Propiedades Psicométricas de la BPNES Adaptada a la Educación Física en Argentina, España y Colombia
}

\author{
Psychometric properties of the BPNES adapted \\ to Physical Education in Argentina, Spain and Colombia
}

\author{
Evelia Franco * 1, Javier Coterón ${ }^{2}$, Valeria Gómez ${ }^{3}$, Jorge Brito ${ }^{4}$ \\ 1 - Universidad Pontificia de Comillas, España. \\ 2 - Universidad Politécnica de Madrid, España. \\ 3 - Universidad de Flores, Argentina. \\ 4 - Universidad de Cuenca, Ecuador.
}

Introducción

Método

Instrumentos

Resultados

Discusión

Referencias

Recibido: 25/07/2017 Revisado: 04/09/2017 Aceptado: 08/09/2017

\section{Resumen}

El objetivo del presente trabajo fue examinar la medida en que las propiedades psicométricas de la Escala de Satisfacción de las Necesidades Psicológicas Básicas (BPNES) resultan estables en muestras de estudiantes de educación física de Argentina, España y Colombia. Participaron 1153 estudiantes de educación física $(44.6 \%$ varones) de edades comprendidas entre 13 y 16 años $(\mathrm{M}=14.68$; $\mathrm{DE}=1.09)$. Los análisis factoriales en muestras independientes revelaron adecuadas propiedades psicométricas del instrumento en los tres países. Los análisis multigrupo posteriores demostraron que la estructura factorial, las cargas factoriales y los interceptos eran invariantes entre los grupos. Sin embargo, se encontraron diferencias entre las varianzas residuales. Se discuten los resultados con base en los hallazgos relativos a la consistencia interna de las dimensiones del instrumento y las diferencias observadas entre las tres muestras. Los hallazgos de este trabajo respaldan posteriores comparaciones interculturales mediante el uso de la BPNES.

Palabras clave: análisis factorial, necesidades psicológicas básicas, invarianza, motivación

\begin{abstract}
The main goal of this study was to examine the extent to which psychometric properties from the Basic Psychological Needs in Exercise Scale (BPNES) are invariant across samples of Argentine, Spanish and Colombian physical education students. The questionnaire was distributed to 1153 students $(44.6 \%$ male) from ages 13 to $16(\mathrm{M}=$ 14.68; $\mathrm{SD}=1.09$ ). Confirmatory factor analyses carried out in independent samples revealed suitable psychometric properties in the three samples. Multi-sample confirmatory factor analyses showed that factor structure, factor loadings and intercepts can be considered invariant across the groups. However, residual variances were found to be different across some of the samples. Findings are discussed in terms of internal consistency and differences observed between the three samples. Findings support valid cross-cultural comparisons using BPNES.
\end{abstract}

Keywords: factor analysis, basic psychological needs, invariance, motivation

*Correspondencia a: Evelia Franco Álvarez, e.francoalvarez@gmail.com

Cómo citar este artículo: Franco, E., Coterón, J., Gómez, V., \& Brito, J. (2017). Propiedades psicométricas de la BPNES adaptada a la educación física en Argentina, España y Colombia. Revista Evaluar, 17(2), 97-112. Recuperado de https://revistas.unc.edu.ar/index.php/revaluar 


\section{Introducción}

A pesar de los innegables beneficios físicos y psicológicos que una vida activa tiene sobre la salud, durante los últimos años se observa un importante descenso del nivel de actividad física (AF) realizada durante la adolescencia (MenesesMontero \& Ruiz-Juan, 2017; Verloigne et al., 2012). Diferentes estudios indican que la educación física $(\mathrm{EF})$ contribuye significativamente a los niveles de práctica de AF en niños y adolescentes (Aibar-Solana et al., 2015); esto es debido a su presencia curricular obligatoria en etapas educativas tempranas de todos los países, lo que facilita un entorno en el que los jóvenes puedan mantener un contacto cotidiano con el deporte y la AF.

La motivación demostrada por los alumnos en el contexto de EF es uno de los factores que más frecuentemente se han relacionado en los últimos años con la práctica deportiva en adolescentes, y es destacable la influencia de la disposición motivacional en los niveles de AF (Standage, Sebire, \& Loney, 2008).

\section{La teoría de la autodeterminación y el rol de las necesidades psicológicas básicas}

La teoría de la autodeterminación (TAD; Deci \& Ryan, 1985) supone un marco teórico ampliamente utilizado en el abordaje de estudios relativos a la motivación en EF (Franco, Coterón, \& Gómez, 2017; Van Aart, Hartman, ElferinkGemser, Mombarg, \& Visscher, 2017).

Según esta teoría, las personas somos organismos activos con una tendencia innata hacia el crecimiento y el desarrollo psicológico; esta se centra en el análisis del grado en que las conductas humanas son autodeterminadas, es decir: la medida en que realizamos las acciones de for- ma voluntaria al nivel más alto de reflexión y nos comprometemos con las mismas con un sentido de elección (Deci \& Ryan, 1985). A través de una de las seis mini-teorías que componen este marco teórico, la teoría de las necesidades básicas, se postula que dicho comportamiento es motivado y regulado por tres necesidades psicológicas primarias y universales: autonomía, competencia y relación con los demás; y la satisfacción de las mismas se considera determinante del grado y tipo de motivación que los individuos presenten.

En el marco de la clase de EF, la necesidad de autonomía considera los esfuerzos de los estudiantes por sentirse el origen de sus acciones y poder determinar su propio comportamiento. La necesidad de competencia se basa en tratar de controlar el resultado y experimentar eficacia en su participación durante las actividades propuestas. Por último, la necesidad de relación con los demás hace referencia a la necesidad de relacionarse y preocuparse por otros y se define mediante dos dimensiones: sentirse aceptado e intimar con los demás (Ryan, 1991).

Las investigaciones indican que cada una de ellas juega un papel importante para el desarrollo y la experiencia óptima y que la satisfacción de las tres necesidades influye positivamente sobre la motivación intrínseca; mientras que la frustración de las mismas se asocia con una menor motivación intrínseca y una mayor motivación extrínseca y desmotivación (Deci \& Ryan, 2000).

Siguiendo los postulados de esta teoría, se han llevado a cabo numerosos estudios relacionados con la AF que demostraron la influencia que la satisfacción de estas necesidades en clase de EF tiene sobre variables relacionadas con la práctica de AF, tanto directamente como indirectamente, a través de la mejora de la motivación autodeterminada (Aibar-Solana et al., 2015; Chatzisarantis \& Hagger, 2009). 
Medición de las necesidades psicológicas básicas en contextos de AF

Con base en lo anteriormente expuesto, se han creado numerosos instrumentos para medir la satisfacción de las necesidades psicológicas básicas (NPB) tales como la Basic Need Satisfaction Scale para contextos de EF (BNS-PE; Ntoumanis, 2005), una adaptación de la escala diseñada por Deci et al. (2001) para contextos laborales, la Psychological Need Satisfaction in Exercise Scale (PNSE; Wilson, Rogers, Rodgers, \& Wild, 2006), o la Escala de Mediadores Motivacionales en el Deporte (EMMD; González-Cutre et al., 2007).

Una de las herramientas más utilizadas en la medida de la satisfacción de las NPB y sus relaciones con la AF es la Basic Psychological Needs in Exercise Scale (BPNES; Vlachopoulos \& Michailidou, 2006). Esta herramienta fue creada y validada para la medición de la satisfacción de las NPB en contextos de ejercicio a partir de los cuestionarios previos de la TAD (Deci \& Ryan, 2001). Se crearon 31 ítems iniciales a partir de los cuales tres expertos del ámbito trabajaron hasta concluir la versión final compuesta por 12 ítems (cuatro para cada una de las necesidades). Los ítems se responden en una escala Likert de 1 (Totalmente en desacuerdo) a 5 (Totalmente de acuerdo). La BPNES ha sido validada en España tanto en el ámbito del ejercicio físico (Sánchez \& Núñez, 2007) como en las clases de EF (MorenoMurcia, González-Cutre-Coll, Chillón-Garzón, \& Parra-Rojas, 2008).

La adecuación de las propiedades psicométricas de la BPNES en diferentes contextos (Cid et al., 2016; Evelein, Korthagen, \& Brekelmans, 2008; Moreno-Murcia, Martínez-Galindo, Moreno-Pérez, Marcos, \& Borges, 2012; Moutao, Serra-Majem, Alves, Leitao, \& Vlachopoulos, 2012; Sánchez \& Núñez, 2007; Vlachopoulos, 2008; Vlachopoulos et al., 2013; Vlachopoulos,
Ntoumanis, \& Smith, 2010) y especialmente el éxito con el que ha sido utilizada en EF (Cuevas, García-López, \& Contreras, 2015; GarcíaGonzález, Aibar, Sevil, Almolda-Tomás, \& Julián-Clemente, 2015; Gómez-Rijo, HernándezMoreno, Martínez-Herráez, \& Gámez, 2014; Méndez-Giménez, Fernández-Río, \& Cecchini, 2013; Sevil, Abós, Julián-Clemente, Murillo, \& García-González, 2015), junto al asequible número de ítems y la redacción clara y sencilla de los mismos, emergen como los principales motivos para trabajar en la adaptación y validación de esta herramienta con el fin de ampliar su uso a diferentes contextos geográficos.

\section{Generalización intercultural}

En tanto que la cultura puede ser una importante influencia sobre diversas variables psicológicas, se hacen necesarios estudios que incorporen análisis interculturales con el fin de evitar la propagación de teorías que puedan no ser generalizables (Duda \& Allison, 1990; Duda \& Hayashi, 1998). Así, en el marco de la TAD, la literatura muestra cómo, si bien existen patrones de comportamiento similares, en ocasiones los constructos principales de la teoría se han relacionado de un modo ligeramente diferente en función del contexto cultural (Cantú-Berrueto, Castillo, López-Walle, Tristán, \& Balaguer, 2016; Pulido, Sánchez-Oliva, González-Ponce, Amado, \& Chamorro, 2017; Van Aart et al., 2017).

En los últimos años, está emergiendo en Latinoamérica una interesante línea de trabajo sobre la motivación en adolescentes, tanto en contextos deportivos (López-Walle, Balaguer, Castillo, \& Tristán, 2011; López-Walle, Tomás, Castillo, Tristán, \& Balaguer, 2011; Reyes, Raimundi, \& Gómez-Correa, 2012) como de EF (Franco, Coterón, Gómez, \& De Franza, 2017; Franco, 
Coterón, Martínez, \& Brito, 2017; Zamarripa, Castillo, Tomás, \& López-Walle, 2016). Estos trabajos han abordado el estudio de las características motivacionales de estos practicantes de actividad física y la relación entre dichas características y diversos patrones comportamentales; $\mathrm{y}$ han encontrado, independientemente del país en el que se realizara el estudio, una relación positiva entre la motivación y patrones más adaptativos en lo que a práctica de actividad física se refiere.

Considerando esta realidad, es necesario tener en cuenta que el uso de un instrumento de medida en un nuevo contexto cultural supone algo más que la mera traducción, administración de cuestionarios y comparación de resultados. En la investigación psicológica resulta de interés la realización de tests de invarianza de instrumentos de medida antes de llevar a cabo análisis de diferencias interculturales (Byrne, 2004), y se deben comprobar las propiedades psicométricas de una escala en los diferentes contextos en que se va a utilizar antes de interpretar las diferencias encontradas (Cheung \& Rensvold, 2002) para garantizar que los ítems de los cuestionarios adquieren el mismo sentido para sujetos de diferentes grupos. En resumen, sin explorar la estructura de los constructos y la adecuación de los ítems para comprobar que en diferentes contextos mantienen el mismo significado, no tendría sentido emprender trabajos de comparación entre países (He \& Van de Vijver, 2012).

Como se ha mencionado anteriormente, la BPNES adaptada a la EF ha mostrado una adecuada validez y confiabilidad en diferentes países iberoamericanos tales como España (MorenoMurcia et al., 2008), Brasil (Moutao et al., 2012), México (Zamarripa et al., 2016) y Portugal (Cid et al., 2016). A pesar de la proliferación de trabajos de la temática desarrollados en países latinoamericanos hispanohablantes, no se han encontrado estudios que evalúen las propiedades psicométricas de este instrumento en Argentina y Colombia. Por lo tanto, el objetivo principal del presente estudio fue analizar las propiedades psicométricas del instrumento con muestras de estos países. Considerando que la BPNES había sido previamente validada en estudiantes de EF españoles, y que la mayor parte de los estudios sobre satisfacción de NPB en contextos hispanohablantes han sido desarrollados en España, se decidió incluir información de adolescentes de este país para, en un segundo paso, evaluar la invarianza de medida del instrumento en estudiantes de Argentina, España y Colombia. Como segundo objetivo, nos planteamos el análisis de posibles diferencias en la satisfacción de las NPB entre los distintos países.

\section{Método}

\section{Participantes}

Se trabajó con un muestreo no probabilístico incidental considerando como criterios de inclusión que los alumnos asistieran a centros educativos ordinarios, participaran en clase de EF con normalidad y tuvieran entre 13 y 16 años de edad. Las respuestas de la BPNES se obtuvieron de 1153 estudiantes de EF (44.6\% varones) de edades comprendidas entre 13 y 16 años (M $=14.68 ; \mathrm{DE}=1.09$ ) de colegios públicos y privados de Argentina $(n=224)$, España $(n=450)$, y Colombia $(n=479)$. En la Tabla 1 se presenta la distribución de la muestra por países, así como los estadísticos descriptivos de género y edad en los participantes de cada país. 
Tabla 1

Distribución de la muestra por país y género, y estadísticos descriptivos de la edad en cada país.

\begin{tabular}{lccccc}
\hline & N total & Hombres & Mujeres & \multicolumn{2}{c}{ Edad } \\
\cline { 5 - 6 } & & & & Rango & M (DE) \\
\hline España & 450 & 205 & 236 & $13-16$ & $14.24(1.05)$ \\
Argentina & 224 & 82 & 142 & $13-16$ & $14.99(.95)$ \\
\hline
\end{tabular}

\section{Instrumentos}

Se utilizó la versión española adaptada a la EF (Moreno-Murcia et al., 2008) de la BPNES (Vlachopoulos \& Michailidou, 2006). La versión utilizada estaba encabezada con el enunciado $E n$ mis clases de EF ... y constaba de 12 ítems, cuatro para medir cada una de las dimensiones (satisfacción de la necesidad de autonomía, satisfacción de la necesidad de competencia y satisfacción de la necesidad de relación con los demás). En la Tabla 2 se presentan los ítems que componen la escala. Dichos ítems se responden en una escala Likert de 1 (Totalmente en desacuerdo) a 5 (Totalmente de acuerdo). Diferentes estudios han proporcionado evidencias de la validez y confiabilidad del instrumento en diferentes contextos (Cid et al., 2016; Moreno-Murcia et al., 2012; Moutao et al., 2012; Sánchez \& Núñez, 2007). Los valores de alfa de Cronbach para la satisfacción de autonomía, competencia y relación con los demás en este estudio fueron respectivamente: $.74, .65$ y .80 en la muestra argentina; $.76, .71$ y .84 en la muestra española, y .77, .71 y .79 en la muestra colombiana.

\section{Diseño y procedimiento}

Se trata de un estudio transversal descriptivo. Los datos fueron recogidos de muestras no probabilísticas de estudiantes de EF de los tres países participantes. Se estableció contacto con los equipos directivos y/o responsables del de- partamento de EF de los diferentes centros de enseñanza para informarles de los objetivos del estudio y pedirles su colaboración. Una vez obtenidas las autorizaciones pertinentes para que los alumnos pudieran participar en el estudio, los cuestionarios fueron administrados durante la clase de EF por un miembro del equipo investigador previamente formado.

Esta persona proporcionó una breve explicación del objetivo del estudio, informó a los participantes sobre la forma de completar el cuestionario e insistió acerca del anonimato de las respuestas solicitando sinceridad a los participantes. El tiempo requerido para completar el cuestionario fue de aproximadamente 15 minutos, variando ligeramente según la edad de los estudiantes. La recolección de datos siguió las indicaciones éticas de la Asociación de Psicología Americana (APA, 2002).

\section{Análisis de datos}

Se examinaron la consistencia interna, con base en el índice de confiabilidad compuesta para cada subescala de la BPNES y el coeficiente alfa de Cronbach; y la estructura factorial a partir de análisis factoriales confirmatorios.

Se consideraron diferentes índices de bondad de ajuste: ratio entre chi-cuadrada y grados de libertad $\left(\chi^{2} / g l\right)$, RMSEA (Root Mean Square Error of Approximation) e índice incremental CFI (Comparative Fit Index). Estos índices de bondad de ajuste son considerados aceptables cuan- 
Tabla 2

Ítems que componen la versión del BPNES administrada (Moreno-Murcia et al., 2008).

\begin{tabular}{ll}
\hline Dimensiones & Ítems \\
\hline & 1. Los ejercicios que realizo se ajustan a mis intereses. \\
Autonomía & 4. La forma de realizar los ejercicios coincide perfectamente con la forma en que yo quiero hacerlos. \\
& 7. La forma de realizar los ejercicios responde a mis deseos. \\
& 10. Tengo la oportunidad de elegir cómo realizar los ejercicios. \\
\hline
\end{tabular}

2. Siento que he tenido una gran progresión con respecto al objetivo final que me he propuesto.

5. Realizo los ejercicios eficazmente.

Competencia

8. El ejercicio es una actividad que hago muy bien.

11. Creo que puedo cumplir con las exigencias de la clase.

3. Me siento muy cómodo/a cuando hago ejercicio con los/as demás compañeros/as.

Relación 6. Me relaciono de forma muy amistosa con el resto de compañeros.

con los demás $\quad 9$. Siento que me puedo comunicar abiertamente con mis compañeros/as.

12. Me siento muy cómodo/a con los/as compañeros/as.

do $\chi^{2} / g l$ presenta valores inferiores a 5 (Bentler, 1989), RMSEA muestra valores por debajo de 0.8 (Browne \& Cudeck, 1993); y el CFI está por encima de 0.9 (Hu \& Bentler, 1995; Marsh, Hau, \& Wen, 2004).

Se utilizó el método de estimación de máxima verosimilitud en base a la matriz de covarianzas junto al procedimiento de bootstrapping, lo que permitió asumir que los datos eran robustos ante la falta de normalidad (Byrne, 2004).

La invarianza se evaluó basándose en un análisis factorial confirmatorio multigrupo. La comparación de modelos fue realizada según la $\triangle \mathrm{CFI}$ y la $\triangle \mathrm{RMSEA}$. Un valor de .01 o menor en la $\triangle$ CFI y de .015 o menor en la $\triangle$ RMSEA indicarían ausencia de diferencia entre los modelos (Chen, 2007; Cheung \& Rensvold, 2002).

Por último, se llevó a cabo un ANOVA para analizar diferencias entre grupos. Se realizó el test de Scheffé para comparar las diferencias entre las puntuaciones de los participantes de los diferentes países.

Se utilizaron los paquetes estadísticos SPSS (IBM, 2016) y AMOS (Arbuckle, 2014) para el análisis de los datos.

\section{Resultados}

El coeficiente de curtosis multivariante de Mardia (50.30) indicó la ausencia de normalidad multivariante (Raykov \& Marcoulides, 2006).

El contraste de la equivalencia factorial comenzó con un análisis preliminar en el que se examinó por separado la bondad de ajuste de la estructura de la BPNES en las muestras de estudiantes de los tres países. Como se muestra en la Tabla 3 , el coeficiente entre el chi cuadrada y los grados 
Tabla 3

Índices de bondad de ajuste del modelo factorial confirmatorio en los tres países.

\begin{tabular}{|c|c|c|c|c|c|c|c|c|c|c|c|}
\hline & \multirow{2}{*}{$\chi^{2}$} & \multirow{2}{*}{$p$} & \multirow{2}{*}{$d f$} & \multirow{2}{*}{$\chi^{2} / g l$} & \multirow{2}{*}{ CFI } & \multirow{2}{*}{ RMSEA } & \multirow{2}{*}{$\begin{array}{l}\text { RMSEA } \\
90 \% \text { IC }\end{array}$} & \multirow{2}{*}{$\begin{array}{c}\text { Cargas } \\
\text { factoriales } \\
\text { estand. }\end{array}$} & \multicolumn{3}{|c|}{$\begin{array}{l}\text { Fiabilidad } \\
\text { Compuesta }\end{array}$} \\
\hline & & & & & & & & & $\mathbf{A}$ & $\mathbf{C}$ & $\mathbf{R}$ \\
\hline Argentina & 87.82 & .001 & 51 & 1.72 & .953 & .057 & $.036-.077$ & $.49-.81$ & .74 & .67 & .80 \\
\hline España & 151.87 & .001 & 51 & 2.98 & .944 & .066 & $.054-.079$ & $.50-.83$ & .74 & .77 & .86 \\
\hline Colombia & 197.30 & .001 & 51 & 3.87 & .919 & .077 & $.066-.089$ & $.48-.81$ & .75 & .77 & .83 \\
\hline
\end{tabular}

de libertad se sitúa por debajo de 5 en todas las muestras. Además, el CFI está por encima de .90 en todos los casos mientras que el RMSEA se encuentra por debajo de .8 en los tres grupos. Según las recomendaciones de Herrero (2010), si un modelo presenta un buen ajuste a través del CFI y del RMSEA conjuntamente, es muy poco probable que el modelo no sea adecuado a los datos.

Tanto los índices de confiabilidad compuesta como el coeficiente alfa de Cronbach resultaron adecuados en líneas generales. Cabe mencionar que los datos de la muestra argentina revelaron una consistencia débil en la dimensión de satisfacción de necesidad de competencia situándose el valor alfa y la confiabilidad compuesta por debajo de los índices recomendados, si bien se decidió interpretar favorablemente los resultados al tratarse de valores cercanos a los establecidos como adecuados.

Las cargas factoriales de los indicadores en sus respectivas variables latentes estuvieron por encima de .50 con excepción del cuarto indicador de competencia en la muestra argentina (Figura 1). El bajo peso de regresión de este indicador sobre su variable latente podría explicar los bajos valores observados en el valor alfa y la confiabilidad compuesta de la dimensión de competencia. Sin embargo, se decidió no suprimir el ítem para evitar la pérdida de información válida para el modelo evaluado (Chin, 1998).
A continuación, se realizaron los análisis multigrupo creando nuevos modelos anidados (Gregorich, 2006) cuyos índices de ajuste se muestran en la Tabla 4. El Modelo 1 (sin restricciones) examinó la invarianza estructural de la BPNES en los tres grupos mostrando adecuados índices de ajuste. Este modelo se consideró como referencia para la subsiguiente anidación de restricciones. El Modelo 2 (restricción de las cargas factoriales) también resultó invariante $(\triangle \mathrm{CFI}<$ .01 ), lo que indica la ausencia de diferencias entre las cargas factoriales de las muestras de Argentina y del resto de los países. El Modelo 3 (en el que se agrega la equivalencia de interceptos) tampoco excede el valor criterio de .01 para $\triangle C F I$ pudiendo aceptarse la hipótesis de invarianza de los interceptos. Los índices de ajuste del Modelo 4 (en el que se agrega la equivalencia de los residuos de los ítems) revelan la ausencia de invarianza residual de las muestras de Argentina con España $(\Delta \mathrm{CFI}=.025)$ y Colombia $(\Delta \mathrm{CFI}=.31)$.

La Tabla 5 muestra la comparación entre las puntuaciones de la muestra argentina y el resto. Los estudiantes argentinos mostraron puntuaciones menores que los participantes españoles en la satisfacción de la necesidad de competencia $\left(\mathrm{M}_{\text {Argentina }}=3.69 ; \mathrm{M}_{\text {España }}=3.83\right)$ y de relación con los demás $\left(\mathrm{M}_{\text {Argentina }}=3.85 ; \mathrm{M}_{\text {España }}=4.15\right)$, no se hallaron diferencias en la satisfacción de autonomía $\left(\mathrm{M}_{\text {Argentina }}=3.15 ; \mathrm{M}_{\text {España }}=3.20\right)$ entre 


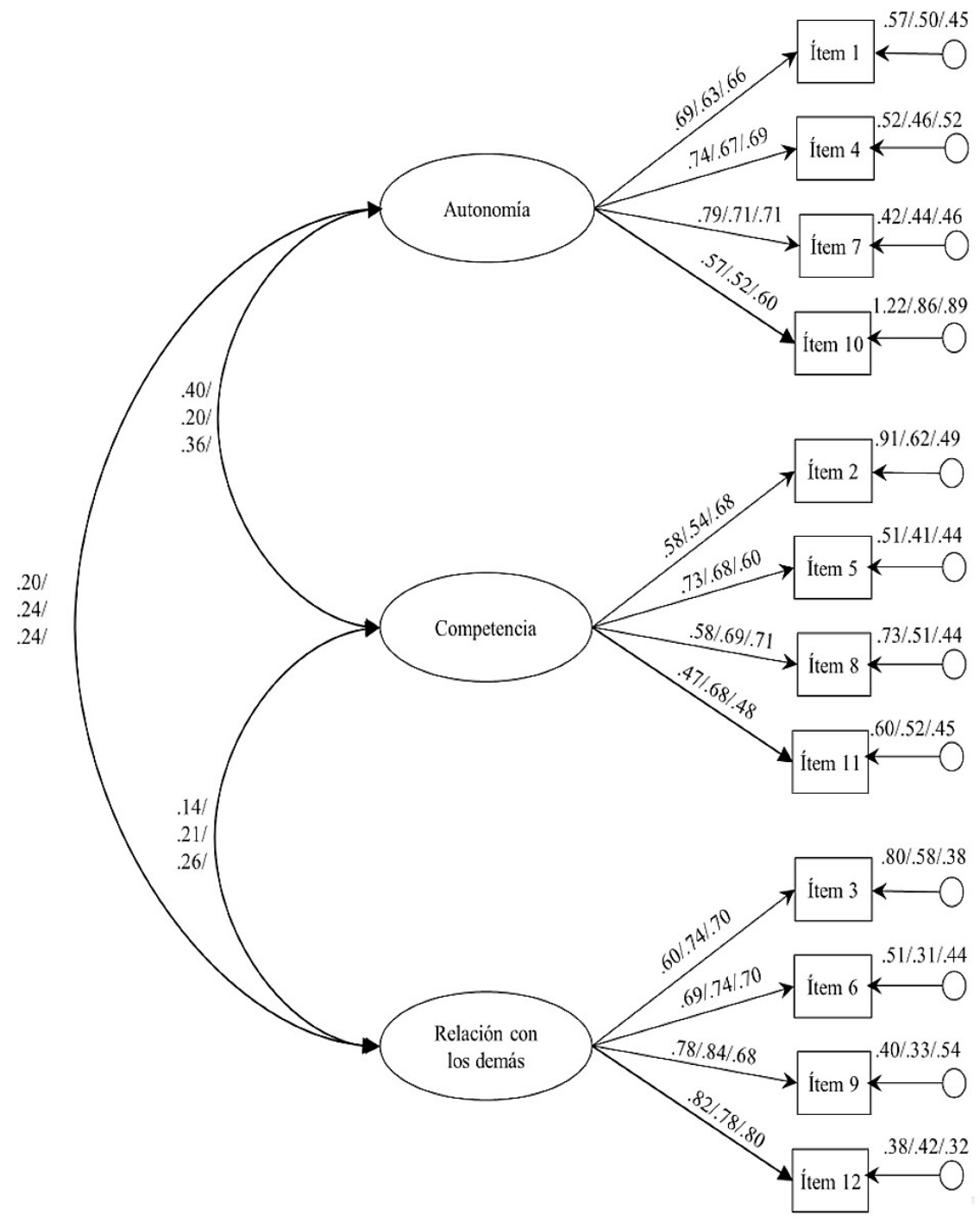

\section{Figura 1}

Parámetros estandarizados (covarianzas entre factores, pesos de regresión y errores de medida) del análisis factorial confirmatorio del BPNES en Argentina, España y Colombia.

Nota. Los parámetros de los diferentes países se presentan separados por / (Argentina/España/Colombia).

estos dos países. Por otra parte, los adolescentes argentinos mostraron menores puntuaciones que los colombianos en la satisfacción de autonomía $\left(\mathrm{M}_{\text {Argentina }}=3.15 ; \mathrm{M}_{\text {Colombia }}=3.48\right)$, de competencia, $\left(\mathrm{M}_{\text {Argentina }}=3.69 ; \mathrm{M}_{\text {Colombia }}=3.89\right) \mathrm{y}$ de relación con los demás $\left(\mathrm{M}_{\text {Argentina }}=3.85 ; \mathrm{M}_{\text {Colombia }}=\right.$ 3.99).

\section{Discusión}

El objetivo del presente estudio fue analizar las propiedades psicométricas y la invarianza de medida de la BPNES en muestras de estudiantes de EF de países diferentes que comparten una misma lengua (Argentina, España y Colombia). 
Tabla 4

Índices de bondad de ajuste de cada uno de los modelos evaluados en la invarianza factorial de la BPNES.

Comparación entre los modelos anidados.

\begin{tabular}{lccccccc}
\hline \multicolumn{1}{c}{ Modelo } & $\chi^{2}$ & $\boldsymbol{g l}$ & CFI & $\Delta$ CFI & RMSEA & $\Delta$ SRMSEA & $\begin{array}{c}\text { RMSEA } \\
\mathbf{9 0 \%} \text { IC }\end{array}$ \\
\hline Argentina - España & & & & & & & \\
Modelo 1 & 239.71 & 102 & .947 & - & .045 & - & $.037-.052$ \\
Modelo 2 & 254.56 & 111 & .944 & .003 & .044 & .001 & $.037-.051$ \\
Modelo 3 & 269.42 & 117 & .941 & .003 & .044 & .000 & $.037-.051$ \\
Modelo 4 & 346.44 & 129 & .916 & .025 & .050 & .006 & $.044-.056$ \\
\hline & & & & & & & \\
\hline Argentina - Colombia & & & & & & & \\
Modelo 1 & 285.11 & 102 & .930 & - & .051 & - & $.044-.058$ \\
Modelo 2 & 293.87 & 111 & .930 & .000 & .048 & .003 & $.042-.055$ \\
Modelo 3 & 312.18 & 117 & .925 & .005 & .049 & .001 & $.042-.055$ \\
Modelo 4 & 404.18 & 129 & .894 & .031 & .055 & .006 & $.049-.061$ \\
\hline
\end{tabular}

Tabla 5

Diferencias entre la muestra argentina y el resto de los países en la satisfacción de las necesidades psicológicas básicas.

\begin{tabular}{|c|c|c|c|c|c|c|c|c|c|}
\hline & \multicolumn{3}{|c|}{ Autonomía } & \multicolumn{3}{|c|}{ Competencia } & \multicolumn{3}{|c|}{ Relación con los demás } \\
\hline & $t$ & $p$ & $d$ Cohen & $t$ & $p$ & $d$ Cohen & $t$ & $p$ & $d$ Cohen \\
\hline Argentina-España & -.81 & .42 & .05 & -2.32 & .020 & .19 & -4.28 & .001 & .35 \\
\hline Argentina-Colombia & -4.76 & .001 & .39 & -3.29 & .001 & .27 & -2.09 & .038 & .17 \\
\hline
\end{tabular}

Esto es, analizar si "el instrumento de medida funciona exactamente de la misma manera y el constructo que está siendo medido tiene la misma estructura teórica para cada grupo de estudio" (Byrne, 2004; p. 272).

El análisis factorial confirmatorio del BPNES desarrollado con cada una de las muestras apoya la estructura del instrumento. De acuerdo con expertos en medición (Cheung \& Rensvold, 2002; Gregorich, 2006), y en línea con trabajos previos que han evaluado la invarianza cultural del BPNES (Cid et al., 2016; Vlachopoulos et al., 2013) así como de otros instrumentos que miden variables motivacionales en entornos de $\mathrm{AF}$
(López-Walle, Tomás, et al., 2011; Wang, Pyun, Kim, \& Chatzisarantis, 2009), se examinó la invarianza estructural a través de un análisis factorial multigrupo.

Los análisis factoriales confirmatorios independientes llevados a cabo con cada una de las muestras utilizadas revelaron que el modelo de medida mostraba unos índices adecuados en los tres países. Las cargas factoriales estandarizadas de cada uno de los indicadores sobre sus factores son aceptables y, en la mayor parte de los casos, aceptadas (Barclay, Higgins, \& Thompson, 1995; Chin, 1998). A pesar de que en términos generales estos pesos de regresión son adecuados, uno de 
los indicadores de la dimensión de competencia muestra una carga factorial muy baja en el análisis factorial llevado a cabo en Argentina y Colombia (.47 y .48 respectivamente). Este indicador se corresponde con el ítem En mis clases de EF pienso que puedo cumplir con las exigencias de la clase. Si consideramos el resto de los ítems utilizados para medir esta dimensión (Siento que he tenido una gran progresión con respecto al objetivo final que me he propuesto; Realizo los ejercicios eficazmente y El ejercicio es una actividad que hago muy bien), se puede comprobar la diferencia existente entre el primer ítem y este último grupo en función del criterio de éxito utilizado para evaluar la competencia (Nicholls, 1984a). Mientras que en los ítems que revelaron una adecuada carga factorial se evidencia un criterio autorreferenciado de éxito (no es necesario que el estudiante se compare con alguien para evaluar su competencia), en el caso del ítem que mostró un bajo peso de regresión la valoración de la competencia se hace en base a un criterio normativo (el éxito del estudiante depende de lo que exija la clase).

Estos datos apuntan a que, si bien la muestra española parece percibir su competencia indistintamente de cuál sea el criterio de referencia, los estudiantes de Argentina y Colombia conceptualizan la competencia de forma de diferente en función del criterio de éxito utilizado. Estudios anteriores que han analizado las percepciones de éxito desde el enfoque de la teoría de las metas de logro (Nicholls, 1984b) en países latinoamericanos y España (Franco, Coterón, \& Martínez, en prensa; López-Walle, Tomás, et al., 2011) han encontrado que los adolescentes de países latinoamericanos puntuaban por encima de los españoles en la orientación al ego, adoptando en mayor medida un criterio normativo de percepción de competencia. Los resultados del presente trabajo, junto a los hallazgos de estos estudios anteriores, sugieren que el escaso peso de regresión del último indicador de satisfacción de competencia podría mejorarse con la modificación de este ítem para que esté en línea con el criterio autorreferenciado presente en los otros tres indicadores.

De la misma manera, y probablemente a consecuencia de lo anteriormente expuesto, la confiabilidad compuesta del factor de competencia en la muestra argentina estuvo por debajo de los límites considerados aceptables (Fornell \& Larcker, 1981). Este resultado refuerza la sugerencia anterior de desarrollar estudios futuros que estudien la confiabilidad del factor de competencia unificando el criterio de éxito en el que se basen los ítems utilizados como indicadores.

En cuanto al análisis multigrupo, los resultados del presente estudio mostraron evidencia de invarianza estructural en todas las muestras. Esto sugiere que los constructos de satisfacción de autonomía, competencia y relación con los demás se conceptualizaron de forma similar en los diferentes grupos, a pesar de la limitación encontrada con el cuarto indicador del factor de competencia. A pesar de que puede existir otro tipo de diferencias entre los países, el contexto cultural no afecta las percepciones de los estudiantes sobre los constructos medidos (Millsap \& Kwok, 2004).

Los resultados también apoyaron la invarianza en las cargas factoriales, lo que significa que los participantes de Argentina, España y Colombia realizaron una interpretación similar de la BPNES y coincidieron en el número de constructos, así como en los ítems asociados a cada constructo (Cheung \& Rensvold, 2002). Según Bollen (1989) la invarianza en las cargas factoriales supone un requisito imprescindible para las comparaciones entre grupos, por lo que los hallazgos del presente estudio pueden sostener futuras investigaciones que busquen comparar la satisfacción de las necesidades psicológicas básicas utilizando la BPNES en estos países.

La invarianza en los interceptos indicó que 
las diferencias interculturales entre las variables latentes indicadas por los ítems son las mismas en los diferentes ítems (Cheung \& Rensvold, 2002), lo que permite llevar a cabo comparaciones entre las muestras de los distintos países tanto de las variables latentes como de las variables observadas.

Los resultados no apoyaron la invarianza en las varianzas residuales de la muestra argentina con la española ni con la colombiana. Basándose en los hallazgos del presente estudio, las comparaciones de las varianzas observadas entre las respuestas obtenidas por los estudiantes argentinos y los españoles o los colombianos no se garantizan. Sin embargo, esto no supone un serio problema ya que las comparaciones de las varianzas no son útiles al comparar resultados de diferentes culturas.

El segundo objetivo del presente estudio fue analizar posibles diferencias entre estudiantes argentinos, españoles y colombianos. Se encontró que los adolescentes argentinos mostraron menor satisfacción de las necesidades de competencia y relación con los demás que los estudiantes españoles, así como puntuaciones inferiores que los adolescentes colombianos en las tres variables.

Existen estudios anteriores que han comparado variables motivacionales frecuentemente asociadas con la satisfacción de las NPB, como la motivación intrínseca o el flow disposicional, en estudiantes de EF de Argentina y España (Franco, Coterón, Gómez, Brito, \& Martínez-González, 2017; Franco, Coterón, Gómez, \& De Franza, 2017). Los resultados de estos estudios han señalado que los adolescentes argentinos tienden a mostrar puntuaciones ligeramente inferiores a las de estudiantes de otros países, lo que acarrea patrones comportamentales menos adaptativos en lo que a práctica de $\mathrm{AF}$ se refiere (Franco, Coterón, Gómez, Brito, et al., 2017).

Si bien no se han hallado estudios que hayan comparado la satisfacción de las NPB entre estudiantes de EF de Argentina y otros países, sí existen en la literatura referencias en las que se ha medido la satisfacción de estas necesidades en Argentina (Franco, Coterón, \& Gómez, 2017) y en España independientemente (MéndezGiménez, et al., 2013). Los resultados de estos trabajos, en línea con los hallazgos del presente estudio, sugieren que si bien las puntuaciones en la variable de satisfacción de autonomía no difieren entre los adolescentes de ambos países, los adolescentes españoles muestran niveles más elevados en la satisfacción de competencia y de relación con los demás. En el trabajo realizado por Franco, Coterón y Gómez (2017) se comprobó la adecuación de un modelo predictivo según el cual las tres necesidades psicológicas jugaban un papel significativo en la predicción de la intención de ser físicamente activo. Los resultados de este estudio sugieren que es relevante fomentar la satisfacción de las NPB en los adolescentes argentinos para mejorar la adherencia a la práctica de actividad física.

En conclusión, los resultados de este estudio apoyan el uso de la BPNES en diversos contextos ofreciendo evidencia de la validación transcultural de la BPNES para utilizarse con estudiantes de EF de tres países que comparten el mismo idioma. Este hallazgo puede facilitar el desarrollo de futuros estudios que examinen las NPB junto a otras variables de actual relevancia. Este trabajo podría considerarse un estudio preliminar de las diferencias interculturales en la satisfacción de las NPB de estudiantes de EF de Argentina, España y Colombia, aunque se hacen necesarias más investigaciones en este ámbito con el fin de expandir el conocimiento sobre las diferencias transculturales en este campo. Por otro lado, los hallazgos del trabajo sugieren que los adolescentes argentinos presentan menor satisfacción con las NPB que los jóvenes españoles y colombianos.

Sería interesante que futuros estudios pro- 
fundizaran en el análisis de la consistencia interna del factor de competencia en la BPNES para su uso en adolescentes latinoamericanos, modificando el ítem que ha mostrado un bajo peso de regresión en la muestra argentina y colombiana y volviendo a comprobar las propiedades psicométricas del instrumento. Por otra parte, parecen necesarias más investigaciones que ahonden en los motivos que expliquen los niveles inferiores en variables motivacionales que muestran los adolescentes argentinos con respecto a los jóvenes de otros países.

Entre las limitaciones del estudio, cabe mencionar la no aleatorización en la selección de la muestra y la ausencia de un análisis de validez concurrente a través del análisis de la asociación de las dimensiones de la BPNES con otros constructos teóricamente relacionados. Por último, es necesario señalar que en las diferencias entre países pudieron haber influido otros factores que no han sido considerados, tales como los contenidos que se trabajan o el estilo interpersonal del profesor.

La principal contribución de este estudio ha sido proporcionar evidencia de la validez y confiabilidad de la versión española de la BPNES para ser utilizada en Argentina y Colombia.

\section{Referencias}

Aibar, A., Julián, J. A., Murillo, B., García-González, L., Estrada, S., \& Bois, J. (2015). Actividad física y apoyo a la autonomía: El rol del profesor de educación física. Revista de Psicología del Deporte, 24(1), 155-161. Recuperado de http://www.rpd-online.com/ article/view/aibar_solana_julian_etal

Arbuckle, J. L. (2014). Amos (Versión 23.0) [software de cómputo]. Chicago: IBM SPSS.

American Psychological Association (2002). Ethical principles of psychologists and code of conduct (Amended
June 2, 2010). American Psychological Association.

Barclay, D., Higgins, C., \& Thompson, R. (1995). The Partial Least Squares (PLS) approach to causal modelling: Personal computer adoption and use as an illustration. Technology Studies, 2(Special Issue on Research Methodology, 2), 285-309.

Bentler, P. M. (1989). EQS structural equations program manual. Los Angeles, CA: BMDP Statistical Software.

Bollen, K. A. (1989). Structural equations with latent variables. New York: Wiley.

Browne, M. W., \& Cudeck, R. (1993). Alternative ways of assessing model fit. En K. A. Bollen \& J. S. Long (Eds.), Testing Structural Equation Models (pp. 136162). Beverly Hills, CA: Sage.

Byrne, B. M. (2004). Testing for multigroup invariance using AMOS: A road less traveled. Structural Equation Modeling, 11(2), 272-300. doi: 10.1207/ s15328007sem1102_8

Cantú-Berrueto, A., Castillo, I., López-Walle, J., Tristán, J., \& Balaguer, I. (2016). Estilo interpersonal del entrenador, necesidades psicológicas básicas y motivación: Un estudio en futbolistas universitarios mexicanos. Revista Iberoamericana de Psicología del Ejercicio y el Deporte, 11(2), 263-270. Recuperado de http://www.webs.ulpgc.es/riped

Cid, L., Lettnin, C., Stobäus, C., Monteiro, D., Davoglio, T., \& Moutão, J. (2016). Cross-cultural validation of the basic psychological needs in Physical Education Scale between Portugal and Brazil samples. Spanish Journal of Psychology, 19(e5), 1-10. doi: 10.1017/ sjp. 2016.6

Chatzisarantis, N., \& Hagger, M. (2009). Effects of an intervention based on self-determination theory on self-reported lesiure-time physical activity participation. Psychology and Health, 24(1), 29-48. doi: 10.1080/08870440701809533

Chen, F. F. (2007). Sensitivity of goodness of fit indexes to lack of measurement invariance. Structural Equation Modeling, 14(3), 464-504. doi: $10.1080 / 10705510701301834$ 
Cheung, G. W., \& Rensvold, R. B. (2002). Evaluating goodness-of-fit indexes for testing measurement invariance. Structural Equation Modeling, 9(2), 233255. doi: 10.1207/S15328007SEM0902_5

Chin, W. W. (1998). Issues and opinion on structural equation modeling. MIS Quarterly, 22(1), 7-17.

Cuevas, R., García-López, L. M., \& Contreras, O. (2015). Influencia del modelo de Educación Deportiva en las necesidades psicológicas básicas. Cuadernos de Psicología del Deporte, 15(2), 155-162. Recuperado de http://revistas.um.es/cpd/

Deci, E. L., \& Ryan, R. M. (1985). Intrinsic motivation and self-determination in human behaviour. New York: Plenum.

Deci, E. L., \& Ryan, R. M. (2000). The "What" and "Why" of goal pursuits: Human needs and the self-determination of behavior. Psychological Inquiry, 11(4), 227-268. doi: 10.1207/s15327965pli1104_01

Deci, E. L., \& Ryan, R. M. (2001). Questionnaires: Basic Psychological Needs Scale. Recuperado de http://selfdeterminationtheory.org/ basic-psychological-needs-scale/

Deci, E. L., Ryan, R. M., Gagné, M., Leone, D., Usunov, J., \& Kornazheva, B. P. (2001). Need satisfaction, motivation, and well-being in the work organizations of a former eastern bloc country: A cross-cultural study of self-determination. PSPB, 27(8), 930-942. doi: $10.1177 / 0146167201278002$

Duda, J. L., \& Allison, M. T. (1990). Cross-cultural analysis in exercise and sport psychology: A void in the field. Journal of Sport and Exercise Psychology, 12(2), 114-131. doi: 10.1123/jsep.12.2.114

Duda, J. L., \& Hayashi, C. (1998). Measurement issues in cross-cultural research within sport and exercise psychology. En J. L. Duda (Ed.), Advances in sport and exercise psychology measurement (pp. 471-483). Morgantown: Fitness Technology.

Evelein, F., Korthagen, F., \& Brekelmans, M. (2008). Fullfillment of the basic psychological needs of student teachers during their first teaching experiences. Teaching and Teacher Education, 24(5), 1137-1148. doi: $10.1016 /$ j.tate.2007.09.001

Fornell, C., \& Larcker, D. F. (1981). Evaluating structural equations models with unobservable variables and measurement error. Journal of Marketing Research, 18, 39-50. doi: 10.2307/3151312

Franco, E., Coterón, J., \& Gómez, V. (2017). El rol de la motivación y la autoestima en la práctica de actividad física en adolescentes. Revista Latinoamericana de Ciencia Psicológica, 9(2), 1-15.

Franco, E., Coterón, J., Gómez, V., Brito, J., \& MartínezGonzález, A. (2017). Influencia de la motivación y del flow disposicional sobre la intención de realizar actividad físico-deportiva en adolescentes de cuatro países. Retos, 31, 46-51. Recuperado de https://recyt. fecyt.es/index.php/retos

Franco, E., Coterón, J., Gómez, V., \& De Franza, A. L. (2017). Relación entre motivación, actividad física realizada en el tiempo libre y la intención futura de práctica de actividad física. Estudio comparativo entre adolescentes argentinos y españoles. SPORT TK, 6(1), 25-34. Recuperado de http://revistas.um.es/ sportk

Franco, E., Coterón, J., \& Martínez, H. A. (en revisión). Invarianza cultural del cuestionario de la orientación a la tarea y al ego y diferencias en las orientaciones motivacionales entre estudiantes de educación física de España, Argentina, Colombia y Ecuador. Revista de Psicología del Deporte.

Franco, E., Coterón, J., Martínez, H. A., \& Brito, J. (2017). Perfiles motivacionales en estudiantes de educación física de tres países y su relación con la actividad física. Suma Psicológica, 24(1), 1-8. doi: 10.1016/j. sumpsi.2016.07.001

García-González, L., Aibar, A., Sevil, J., Almolda-Tomás, F. J., \& Julián-Clemente, J. A. (2015). Soporte de autonomía en educación física: Evidencias para mejorar el proceso de enseñanza. Cultura, Ciencia y Deporte, 10(29), 103-111. doi: 10.12800/ccd.v10i29.547

Gómez-Rijo, A., Hernández-Moreno, J., Martínez-Herráez, I., \& Gámez, S. (2014). Necesidades psicológicas básicas en educación física según el género y el curso 
del estudiante. Revista de Investigación Educativa, 32(1), 159-167. doi: 10.6018/rie.32.1.172311

González-Cutre, D., Martínez-Galindo, C., AlonsoVillodre, N., Cervelló-Gimeno, E., Conte-Marín, L., \& Moreno-Murcia, J. A. (2007). Las creencias implícitas de habilidad y los mediadores psicológicos como variables predictoras de la motivación autodeterminada en deportistas adolescentes. En J. Castellano \& O. Usabiaga (Eds.), Investigación en la Actividad Física y el Deporte II (pp. 407-417). Vitoria: Universidad del País Vasco.

Gregorich, S. E. (2006). Do self-report instruments allow meaningful comparisons across diverse population groups? Testing measurement invariance using the confirmatory analysis framework. Medical Care, 44(Suppl 3), S78-S94. doi: 10.1097/01. mlr.0000245454.12228.8f

He, J., \& Van de Vijver, F. (2012). Bias and equivalence in cross-cultural research. Online Readings in Psyhology and Culture, 2(2). doi: 10.9707/2307-0919.1111

Herrero, J. (2010). El análisis factorial confirmatorio en el estudio de la estructura y estabilidad de los instrumentos de evaluación: Un ejemplo con el Cuestionario de Autoestima CA-14. Psychosocial Intervention, 19(3), 289-300. doi: 10.5093/in2010v19n3a9

Hu, L., \& Bentler, P. M. (1995). Evaluating model fit. En R. Hoyle (Ed.), Structural equation modeling: Issues, concepts, and applications. Newbury Park, CA: Sage.

IBM Corp. (2016). IBM SPSS Statistics for Windows, Version 24.0. [software de cómputo] Armonk, NY: IBM Corp.

López-Walle, J., Balaguer, I., Castillo, I., \& Tristán, J. (2011). Clima motivacional percibido, motivación autodeterminada y autoestima en jóvenes deportistas mexicanos. Revista de Psicología del Deporte, 20(1), 209-222. Recuperado de http://www.redalyc. org/html/2351/235119302015/

López-Walle, J., Tomás, I., Castillo, I., Tristán, J., \& Balaguer, I. (2011). Invarianza factorial del TEOSQ en jóvenes deportistas mexicanos y espa- ñoles. Revista Mexicana de Psicología, 28, 53-61. Recuperado de http://eprints.uanl.mx/5312

Marsh, H. W., Hau, K. T., \& Wen, Z. (2004). In search of golden rules: Comment on hypothesis-testing approaches to setting cutoff values for fit indexes and dangers in overgeneralizing Hu and Bentler's (1999) findings. Structural Equation Modeling, 11(3), 320341. doi: 10.1207/s15328007sem1103_2

Méndez-Giménez, A., Fernández-Río, J., \& CecchiniEstrada, J. A. (2013). Papel importante del alumnado, necesidades psicológicas básicas, regulaciones motivacionales y autoconcepto físico en educación física. Cuadernos de Psicología del Deporte, 13(1), 71-82. doi: 10.4321/s1578-84232013000100008

Meneses-Montero, M., \& Ruiz-Juan, F. (2017). Estudio longitudinal de los comportamientos y el nivel de actividad físico-deportiva en el tiempo libre en estudiantes de Costa Rica, México y España. Retos, 31, 219-226. Recuperado de https://dialnet.unirioja.es/ servlet/articulo? codigo $=5841375$

Millsap, R. E., \& Kwok, O. (2004). Evaluating the impact of partial factorial invariance on selection in two populations. Psychological Methods, 9(1), 93-115. doi: 10.1037/1082-989X.9.1.93

Moreno-Murcia, J. A., Martínez-Galindo, C., MorenoPérez, V., Marcos, P. J., \& Borges, F. (2012). Confirmation of the Basic Psychological Needs in Exercise Scale (BPNES) with a sample of people who do healthy exercise. Journal of Sports Science and Medicine, 11, 141-146. Recuperado de http:// www.jssm.org

Moreno-Murcia, J. A., González-Cutre-Coll, D., ChillónGarzón, M., \& Parra-Rojas, N. (2008). Adaptación a la educación física de la Escala de las Necesidades Psicológicas Básicas en el Ejercicio. Revista Mexicana de Psicología, 25(2), 295-303. Recuperado de http://www.redalyc.org/pdf/2430/243016308009. pdf

Moutão, J., Serra-Majem, L., Alves, J. A., Leitao, J. C., \& Vlachopoulos, S. (2012). Validation of the Basic Psychological Needs in Exercise Scale in a 
Portuguese sample. Spanish Journal of Psychology, 15(1), 399-409. doi: 10.5209/rev_sjop.2012.v15. $\mathrm{n} 1.37346$

Nicholls, J. G. (1984a). Achievement motivation: Conceptions of ability, subjective experience, task choice, and performance. Psychological Review, 91(3), 328-346. doi: 10.1037/0033-295x.91.3.328

Nicholls, J. G. (1984b). Conceptions of ability and achievement motivation. En R. E. Ames \& C. Ames (Eds.), Research on motivation in education, Vol. 1: Student motivation (pp. 39-73). New York: Academic Press.

Ntoumanis, N. (2005). A prospective study of participation in optional school physical education using a Self-Determination Theory framework. Journal of Educational Psychology, 97(3), 444-453. doi: 10.1037/0022-0663.97.3.444

Pulido, J. J., Sánchez-Oliva, D., González-Ponce, I., Amado, D., \& Chamorro, J. L. (2017). Adaptación al fútbol de la Escala de Necesidades Psicológicas Básicas en el Ejercicio. Revista Iberoamericana de Psicología del Ejercicio y el Deporte, 11(2), 177184. Recuperado de http://www.redalyc.org/articulo. oa?id=311145841001

Raykov, T., \& Marcoulides, G. A. (2006). A first course in structural equation modeling. Mahwah, $\mathrm{NJ}$ : Erlbaum.

Reyes, M., Raimundi, M. J., \& Gómez-Correa, L. (2012). Programa de entrenamiento en habilidades psicológicas en jugadoras de voleibol de alto rendimiento. Cuadernos de Psicología del Deporte, 12(1), 9-16. doi: 10.4321/s1578-84232012000100001

Ryan, R. M. (1991). The nature of the self in autonomy and relatedness. En J. Strauss \& G. R. Goethals (Eds.), Multidisciplinary perspectives on the self (pp. 208238). New York: Springer-Verlag.

Sánchez, J., \& Núñez, J. L. (2007). Análisis preliminar de las propiedades psicométricas de la versión española de la Escala de Necesidades Psicológicas Básicas en el Ejercicio Físico. Revista Iberoamericana de Psicología del Ejercicio y el Deporte, 2(2), 83-92. Recuperado de http://www.redalyc.org/articulo. oa? $\mathrm{id}=311126258006$

Sevil, J., Abós, A., Julián-Clemente, J. A., Murillo, B., \& García-González, L. (2015). Género y motivación situacional en educación física: Claves para el desarrollo de estrategias de intervención. Revista Internacional de Ciencias del Deporte, 11(41), 281296. doi: 10.5232/ricyde2015.04106

Standage, M., Sebire, S. J., \& Loney, T. (2008). Does exercise motivation predict engagement in objectively assessed bouts of moderate-intensity exercise?: A Self-Determination Theory perspective. Journal of Sport and Exercise Phychology, 30(4), 337-352. doi: 10.1123/jsep.30.4.337

Van Aart, I., Hartman, E., Elferink-Gemser, M., Mombarg, R., \&Visscher, C. (2017). Relations among basic psychological needs, PE-motivation and fundamental movement skills in 9-12-year-old boys and girls in Physical Education. Physical Education and Sport Pedagogy, 22(1), 15-20. doi: 10.1080/17408989.2015.1112776

Verloigne, M., Lippevelde, W. V., Maes, L., Yildrim, M., Chinapaw, M., Manios, Y. ... De Bourdeaudhuij, I. (2012). Levels of physical activity and sedentary time among 10- to 12- year-old boys and girls across 5 European countries using accelerometers: An observational study within the ENERGY-project. International Journal of Behavioral Nutrition and Physical Activity, 9(1), 34. doi: 10.1186/1479-5868-9-34

Vlachopoulos, S. (2008). The Basic Psychological Needs in Exercise Scale: Measurement invariance over gender. Structural Equation Modeling, 15(1), 114-135. doi: 10.1080/10705510701758398

Vlachopoulos, S., Asci, F. H., Cid, L., Ersoz, G., González-Cutre, D., Moreno-Murcia, J. A., \& Moutao, J. (2013). Cross-cultural invariance of the Basic Psychological Needs in Exercise Scale and need satisfaction latent mean differences among Greek, Spanish, Portuguese and Turkish samples. Psychology of Sport \& Exercise, 14(5), 622-631. doi: 10.1016/j.psychsport.2013.03.002 
Vlachopoulos, S., \& Michailidou, S. (2006). Development and initial validation of a measure of autonomy, competence and relatedness: the Basic Psychological Needs in Exercise Scale. Measurement in Physical Education \& Exercise Science, 10(3), 179-201. doi: 10.1207/s15327841mpee1003_4

Vlachopoulos, S., Ntoumanis, N., \& Smith, A. (2010). The Basic Psychological Needs in Exercise Scale: Translation and evidence for cross-cultural validity. International Journal of Sport and Exercise Psychology, 8(4), 394-412. doi: 10.1080/1612197x.2010.9671960

Wang, J. C. K., Pyun, D. Y., Kim, J. Y., \& Chatzisarantis, N. (2009). Testing for multigroup invariance of the perceived locus of causality in sport. Personality and Individual Differences, 47(6), 590-597. doi: 10.1016/j.paid.2009.05.008

Wilson, P., Rogers, W., Rodgers, W., \& Wild, T. (2006). The Psychological Need Satisfaction in Exercise Scale. Journal of Sport and Exercise Phychology, 28(3), 231-251. doi: 10.1123/jsep.28.3.231

Zamarripa, J., Castillo, I., Tomás, I., \& López-Walle, J. (2016). Validación mexicana del Cuestionario de Apoyo a las Necesidades Psicológicas Básicas en la Educación Física. Revista Mexicana de Psicología, 33(2), 143-150. 